\title{
Aplicación de la contametría como tecnología social en casos de desastres ambientales provocados por el hombre. Una aproximación *
}

Recibido: 17 de julio de 2014 • Aceptado: 7 de septiembre de 2014.

\section{Application of Accountametrics as a Social Technology in Cases of Environmental Man-Made Disasters. An Approach}

Application de la comptamétrie comme technologie sociale en cas de catastrophes écologiques d'origine humaine. Une approche

Aplicaçáo da métrica contábil como tecnologia social em casos de catástrofes ambientais provocadas pelo homem. Uma aproximaçáo

Amparo Elizabeth Pérez Pita ${ }^{a}$

Rosa Trujillo Rendón ${ }^{b}$

* Artículo resultado del proyecto de investigación titulado "Aplicaciones contamétricas", inscrito en la línea de Investigación Sistemas de Información Contable y Control, de la Facultad de Ciencias Económicas, Administrativas y Contables de la Universidad Libre, sede Bogotá, Colombia.

a Contadora Pública, Especialista en Gestión Tributaria. Especialista en Gerencia Financiera Internacional y estudiante de Maestría en Contabilidad, Universidad Libre. Docente de la Universidad Libre y de la Universidad Tecnológica de Bolívar. Comentarios a: amparo.perezp@unilibrebog.edu.co

b Contadora Pública de la Universidad de la Salle, Especialista en Impuestos, Magíster en Docencia y estudiante de Maestría en Contabilidad. Docente de la Universidad Libre. Comentarios a: rosa.trujillor@ unilibrebog.edu.co 
Resumen. El objetivo del presente trabajo es hacer una aproximación contamétrica de la responsabilidad socioambiental del contador público para clasificar, medir, valorar, revelar, interpretar, evaluar y controlar el impacto generado por los desastres ambientales. Tales factores se conciben aquí como los elementos contamétricos en el sentido de Franco (2014). Para ello se tomó el caso de una empresa explotadora de carbón que ocasionó el conocido desastre ambiental del 13 de enero del 2013, debido al vertimiento de este mineral en la Ciénaga Grande de Santa Marta, Colombia. En el diseño metodológico se consultaron fuentes documentales primarias (caso Drummond) y secundarias (artículos especializados en la materia). Se concluye que los resultados todavía son preliminares, dado que la contametría es un saber en construcción. Sin embargo, se espera que para el desarrollo de investigaciones futuras el caso pueda extrapolarse a otros similares o susceptibles de ser comparados.

Palabras clave: contametría, desastres ambientales, caso Drummond, elementos contamétricos, responsabilidad socioambiental.

Abstract. The purpose of this paper is a countermetrical approaching to the environmental responsibility of the public accountant to classify, measure, assess, disclose, interpret, evaluate, and control the environmental impact from disasters. Taking into account what Franco says (2012), we think of those factors as countermetrical elements. To do so, we studied the case of a coalmining company that caused the well-known environmental disaster occurred on January $13^{\text {th }}, 2013$, due to the dumping of this mineral into the Ciénaga Grande de Santa Marta, Colombia. For the methodological design we consulted several primary sources (Drummond case) as well as secondary (specialized articles on the subject). We conclude that the findings are still preliminary, since Countermetrics is an emerging knowledge. However, it is expected that further research could extrapolate this case to other similar or likely to be compared.

Keywords: Countermetric elements, environmental responsibility, Countermetrics, Drummond case, environmental disasters.

Résumé. Le but de ce papier est de rapprocher la responsabilité environnementale Contamétrica du comptable public de le classer, mesurer, évaluer, communiquer, interpréter, évaluer et maîtriser l'impact environnemental des catastrophes générées. Ces facteurs sont conçus ici comme des éléments de contamétricos, dans ce Franco (2014). Cela a pris le cas d'un exploitant de charbon qui a causé la catastrophe écologique comme avant 13 Janvier, 2013, en dumping de ce minéral dans la Cienaga Grande de Santa Marta, en Colombie. La conception méthodologique primaires sources documentaires secondaires (si Drummond) et (articles scientifiques sur le sujet) ont été consultés. Nous concluons que les résultats sont encore préliminaires, depuis le contametría est la construction de la connaissance. Cependant, on s'attend à ce que la recherche dans le développement futur de la charge ne peut être extrapolés à d'autres semblables ou susceptibles d'être comparées.

Mots-clés: Les éléments de contamétricos; la responsabilité environnementale; contametría; Drummond; catastrophes environnementales.

Resumo. O objectivo deste trabalho para aproximar a responsabilidade ambiental Contamétrica do contador público para classificar, medir, avaliar, divulgar, interpretar, avaliar e controlar o impacto ambiental desastres gerados. Tais fatores são concebidos aqui como os elementos contamétricos, em que Franco (2014). Isso levou o caso de um operador de carvão que causou o desastre ambiental conhecido 13 de janeiro de 2013 pelo dumping deste mineral na Ciénaga Grande de Santa Marta, na Colômbia. O desenho metodológico fontes documentais primárias secundárias (se Drummond) e (artigos acadêmicos sobre o assunto) foram consultados. Conclui-se que os resultados ainda são preliminares, uma vez que o contametría é a construção do conhecimento. No entanto, espera-se que futuras pesquisas em desenvolvimento do caso podem ser extrapolados para outros semelhantes ou que possam ser comparados.

Palavras-chave: contamétricos elementos; responsabilidade ambiental; contametría; Drummond; desastres ambientais. 


\section{Antecedentes}

En las últimas décadas se han venido conformando movimientos socioambientales en el mundo debido a la preocupación por la protección del medio ambiente, por la necesidad de toma de conciencia y por la imperante obligación de cuidar lo que nos queda de él y de fomentar un desarrollo económico que no agreda más al planeta. Esta preocupación ha inquietado paulatinamente a los gobiernos de algunos países, los cuales, a través de compromisos de carácter internacional, ${ }^{1}$ han venido desarrollando políticas internas de control y de protección ambiental. Estas acciones se han propuesto porque, ciertamente, cada vez es más difícil pasar por alto el peligro inminente que significa el desequilibrio y el perjuicio, en muchos casos irremediable, que el desarrollo económico ha generado y sigue generando en el medio ambiente.

Para explicar de algún modo este panorama desolador, se puede decir que la pobreza, sinónimo de miseria humana, afecta grandes masas de la población; esto se traduce en más gente y menos tierra, lo que lleva al agotamiento de los recursos naturales y a la destrucción de la biodiversidad. Así, ante la falta de un empleo consciente de los recursos, ante el consumo excesivo de combustible fósil, el "efecto invernadero", el agotamiento de la capa de ozono, la contaminación sistemática, el menor crecimiento económico y el menor bienestar humano, inevitablemente volvemos al punto de partida: la creciente pobreza (Zapata, 1997, p. 17).

Es claro que los problemas ambientales siguen creciendo y han dejado de ser problemas locales para convertirse en una preocupación global, lo que lleva a pensar en la supervivencia básica de la humanidad. La población humana actual sobrepasa los 7,2 billones y se espera que alcance 8,5 billones para 2025, fecha que está bastante cerca cuando aún no se han tomado medidas de fondo al respecto. Por el egoísmo de los grandes contaminadores no hay un acuerdo para disminuir o controlar el desarrollo económico, ni para disminuir las terribles consecuencias que estamos viviendo y, que a corto plazo, serán nefastas si no se toma la decisión de empezar a corregir el comportamiento y la actitud hacia la naturaleza que está bastante resentida. ${ }^{2}$

\footnotetext{
${ }^{1}$ Hacia los años setenta ya se comenzaban a vislumbrar las consecuencias del maltrato a la naturaleza, por lo que tuvo lugar la "Declaración de Estocolmo" en 1972, que proclamaba la necesidad de equilibrar el desarrollo económico de la humanidad y la protección del medio ambiente. El 16 de septiembre de 1987 es firmado el protocolo de Montreal, que tuvo como objetivo firmar un acuerdo internacional para controlar la producción de sustancias que se creen responsables del agujero en la capa de ozono. En Río de Janeiro, entre el 3 y el 14 de junio de 1992, la comunidad internacional aprobó el convenio sobre la diversidad biológica con el fin de prevenir la reducción o pérdida significativa de la diversidad por la deforestación y tala indiscriminada de árboles. El 11 de diciembre de 1997 se firmó en Kyoto un documento titulado El Protocolo de Kyoto, sobre el cambio climático, instrumento que se creó con el fin tomar medidas para reducir los gases de efecto invernadero. En esta reunión los países participantes se comprometieron a reducir en un 5,2\% las emisiones contaminantes (desde el 2008 hasta el 2012), tomando como punto de partida las concentraciones de contaminación por emisión de estos gases durante 1990. Este acuerdo entró en vigor en febrero de 2005, después de que Rusia aceptara el compromiso. Siguiendo la preocupación de la comunidad mundial, tuvo lugar una nueva reunión en Johannesburgo entre el 26 de agosto y el 4 de septiembre de 2002, treinta años después de la conferencia de Estocolmo y 20 años después de la reunión de Río de Janeiro. Sin embargo, según los entendidos, esta reunión se hace demasiado tarde para impedir los problemas que se pretendía resolver, pues se ignora el principio de precaución; pero también tiene lugar muy pronto para conseguir acuerdos satisfactorios y significativos, ya que a pesar de los tratados anteriores ya mencionados y de las demás reuniones en los ámbitos regional y local, los problemas sociales y ambientales se han agravado debido al crecimiento desordenado de la población y a la pobreza extrema en que viven 800 millones de personas

2 De acuerdo con el Instituto Mundial de Recursos, si la tasa de crecimiento del 3 \% de la actividad económica mundial (que se viene dando desde los ańos cincuenta) continúa, para 2050 será cinco veces mayor y, para entonces, no habrá recursos naturales suficientes para sostener al mismo tiempo la población que, como ya se dijo, también crece de una manera desorganizada.
} 
Como si lo anterior no fuera suficiente, y para demostrar que no es exagerado hablar de peligro inminente, son destruidos 17 millones de hectáreas de bosque y pastura por ańo, lo que disminuye notablemente el agua dulce y la biodiversidad, fuertemente amenazada en los últimos 100 años. La problemática ha sido y es foco de muchas discusiones de tipo científico, académico y político. Hoy proliferan propuestas, estudios, escritos y documentales, entre otros, que muestran diferentes aspectos del panorama actual y, al mismo tiempo, las perspectivas y posibles soluciones que van desde las más radicales, como el crecimiento cero y la aplicación estricta del principio de precaución, ${ }^{3}$ hasta la despreocupación total por el medio ambiente ante la imposibilidad de frenar el modelo de desarrollo económico capitalista y de controlar la entropía ambiental.

Entre las más importantes propuestas no extremistas se encuentra la del desarrollo sostenible, que plantea la posibilidad de progresar en armonía con la naturaleza, es decir, hacer compatible la preservación del medio ambiente con el crecimiento económico. Ciertamente se trata de un ideal muy difícil de llevar a la práctica de manera cabal, pero que, por lo menos, mantiene el equilibrio de intereses fundamentales de la humanidad y ofrece alternativas viables para el presente. Sin embargo, para cumplir lo que se pretende con la idea del desarrollo sostenible, el mundo globalizado debe ir más allá de reconocer la interdependencia económica; debe comprender y aceptar la interdependencia ecológica y, aún más, el "ensamble” de las dos (MacNeill, Winsemius y Yakushji, 1991).

La perspectiva en el ámbito global, si deseamos sobrevivir en armonía, debe ser la fusión de lo ecológico y lo económico. Es claro que no basta con regular las relaciones sociales entre hombres, sino que se hace indispensable la regulación de la relación entre hombre y medio ambiente, pues, de acuerdo con Edgar Morin y Fritjof Capra, podemos decir que hombres y medio ambiente son uno solo (citados por Conceicao De Almeida, 2008).

Esta propuesta, a su vez, tiene tres facetas que no se deben perder de vista cuando se trata de mejorar el medio ambiente. Primero, los temas que tienen que ver con el medio ambiente se pueden cuantificar en términos económicos, es decir, se tiene la posibilidad de identificar con cifras el nivel de la degradación y la destrucción ambiental. En segundo lugar, una vez identificado el problema se pueden utilizar las herramientas e instrumentos económicos para alcanzar las metas ambientales tales como la reducción y, posteriormente, el control de la contaminación; por último, ser conscientes del alcance de estas herramientas en cuanto al control de lo ambiental, puesto que está demostrando que hay limitaciones para medir los daños ambientales (Zapata Lugo, 1997).

La discusión de la dimensión ecológica del desarrollo sostenible ha demostrado que los modelos de desarrollo aplicados han dado como resultado un grave menoscabo del medio ambiente, ya que los procesos industriales o de producción no tienen en cuenta los efectos colaterales que ocasionan, además de la falta de cuantificación de los gastos ambientales, entre otros. Estos factores son los grandes responsables de la degradación y el agotamiento silencioso del medio ambiente soportados por la sociedad. Según lo dicho, se deben tomar medidas extremas, ya que, muy seguramente, tendrían efectos económicos no deseables para unos pocos, pero que brindarían aire más puro, agua potable y esperanza para la humanidad.

\footnotetext{
3 Suspender toda actividad de explotación económica hasta que no se demuestre que no es nociva para el medio ambiente.
} 
Ronald Harry Coase, Premio Nobel de Economía en 1960, publicó un artículo titulado "The problem of social cost"; en él propone que la solución no es "el que contamina paga", sino que se puede optar por no contaminar, prever los efectos colaterales en el medio ambiente, cumplir con todas las normas ambientales utilizando tecnología limpia y buscando otras alternativas que no cobren el precio tan alto que significa destruir la naturaleza (Coase, 1988). Colombia no es ajena a esta problemática, pues forma parte de la Tierra y del cosmos y, al igual que los demás países en vía de desarrollo y de los desarrollados, tiene grandes problemas ambientales creados por los grandes, medianos y pequeños productores que generan gran contaminación y destrucción del medio ambiente sin ningún control, aparte de los graves problemas ecológicos mundiales que, en su mayoría, son causados por los países desarrollados. No obstante, más allá de las condiciones y compromisos globales ${ }^{4}$ que se deberían presentar para poder hablar de desarrollo sostenible en Colombia, y que escapan al objeto de este escrito, hay medidas internas que Colombia está en la obligación y posibilidad de tomar, algunas de las cuales se han tomado, dadas las nuevas preocupaciones que encara la Constitución Política de 1991, y la creación del Ministerio del Medio Ambiente.

La Constitución Nacional de Colombia, en el artículo 79, sobre los Derechos colectivos y del ambiente, garantiza a toda persona el derecho a gozar de un ambiente sano y el consecuente deber del Estado de proteger la diversidad e integridad del ambiente. Después, el artículo 80 plantea el desarrollo sostenible como el camino de equilibrio entre la conservación y explotación de los recursos naturales. Dadas estas nuevas implicaciones del problema ambiental, y en vista de la alternativa de desarrollo sostenible por la cual opta la legislación nacional, la protección del medio ambiente se vuelve una cuestión que compete de manera coordinada al gobierno — a través de la legislación y el control y promoción de los principios de protección del medio ambiente- y a los particulares - tales como promotores y ejecutores de los principios y normas sobre el medio ambiente-, desde las diferentes profesiones, oficios y actividades económicas.

De este modo, vemos que, por ejemplo, la Ley 99 de 1993, por la cual se crea el Ministerio de Medio Ambiente - hoy Ministerio de Medio Ambiente y Desarrollo Sostenible_-, da cuenta de la obligación del componente legislativo en un Estado social de derecho como el colombiano; además, esta ley contiene importantes disposiciones, algunas de las cuales atañen al campo contable, cuyo desarrollo tendría consecuencias de gran relevancia y efecto. En este sentido, debe tenerse en cuenta que cualquier gobierno en su repertorio de soluciones tiene varias herramientas entre las cuales se encuentran las regulaciones económicas, los mecanismos de mercado y los autorreguladores. La regulación económica corresponde a la intervención gubernamental, como el establecimiento de impuestos, tasas, contribuciones, entre otras, para que a través de ellos se limiten, controlen o mitiguen los daños ambientales. Los mecanismos de mercado proponen que las empresas productoras, en general, reflejen en sus presupuestos y estados financieros los costos del deterioro ambiental. Esto significa que las grandes industrias den un primer paso al incluir en sus

4 Una de las variables globales más determinantes es la difícil relación a la que se ha llamado "sur contra norte," debido crisis de la deuda que ha limitado las posibilidades de un crecimiento económico sostenible en los países en vía de desarrollo. Por eso los temas ambientales deben ser tratados globalmente, para que las condiciones sean equilibradas entre ambos bandos. A esto se agrega que son los países industrializados los que más contaminan y los que más están consumiendo los recursos materiales de los países en vía de desarrollo, mientras, con frecuencia, transfieren sus desechos a los países subdesarrollados. 
finanzas los costos ambientales y la autorregulación que estaría en armonía con la ética o moralidad de las corporaciones, las cuales deben tomar conciencia y adoptar mecanismos complementarios para proteger el medio ambiente (Zapata Lugo, 1997). Es aquí donde el contador público debe asumir su responsabilidad social, de tal forma que represente en los estados financieros no solamente los hechos que tienen que ver con los intereses económicos del inversionista, sino todos los que afectan el medio ambiente, hechos que hasta el momento se han pasado por alto, no solo por parte de los estamentos del estado sino por los profesionales que han sido educados únicamente para valorar lo que significa utilidad de manera inmediata, entre ellos los contadores públicos.

\section{Contametría: herramienta para el contador público}

En el caso concreto del contador público, quien aparentemente pareciera ajeno o sin herramientas para contribuir en la solución de la problemática, se encuentran grandes posibilidades de aportes trascendentales que lo hacen desempeñar un papel determinante por medio de la contametría, herramienta que le permite al contador clasificar, representar, medir, valorar, interpretar, evaluar y controlar todos los hechos económicos que estén relacionados con la empresa y, en particular, los efectos de esta en el medio ambiente. La contametría le permite al contador público informar sobre los hechos sociales que la contabilidad financiera no tiene en cuenta por no estar dentro de sus intereses inmediatos, pero que el contador público, siendo fiel a su deber, debe empezar a reflejar en sus informes para generar conciencia social y respeto por la naturaleza que a todos pertenece.

La contametría es un concepto joven que solamente lleva unos 27 ańos de ser mencionado en discursos académicos, pero que empieza a ser desarrollado en América Latina. Se trata de una tecnología para la contabilidad cimentada a partir de bases teóricas de la contabilidad o contadología (Franco, 2014). Para que sea tomada en serio requiere de bases que determinen que la contabilidad es una sola y no la sumatoria de modelos especializados postulados por la administración. Esta herramienta cuenta con cuatro dimensiones: instrumental, social, temporal y praxeológica (Franco, 2014), que permiten visualizar desde otra óptica que no es exactamente la financiera, como ya se ha mencionado, sino que muestra los hechos sociales de una forma más integral.

La dimensión instrumental reúne procesos relacionados con la clasificación, medición, valoración, revelación, interpretación, evaluación y control de eventos o hechos sociales; la social abarca factores de identidad en cuanto al territorio, cultura, población y economía; la temporal implica el análisis del pasado, el presente y el futuro de los hechos sociales, y la praxeológica se relaciona con exámenes críticos de los factores morales, presentes en los hechos informados contablemente.

La finalidad de la contametría es solucionar las incoherencias tradicionales de la contabilidad como son la división cognitiva, la unión o mezcla de medición y valoración, las fusiones de dimensión de la información y los sesgos hacia los intereses de ciertos grupos en perjuicio de otros. La contametría propone que las representaciones sociales derivadas de los hechos económicos y sociales de las empresas estén fundamentadas en intereses y valores de todos los grupos interesados. 
De acuerdo con el profesor Franco, a pesar de que la contametría es un concepto que hasta ahora está en construcción, permite dimensionar de una manera más integral aquellos hechos sociales que siempre han sido ignorados con la disculpa de no poder ser caracterizados y que todos sabemos deben ser representados desde la contabilidad. Estos elementos aportados por la contametría, y que son útiles para darle una mano al medio ambiente, patrimonio de todos, son:

1. La clasificación: elemento capital de la información, está constituido por aspectos cualitativos y cuantitativos. Se considera como un grupo de elementos a partir de las propiedades compartidas, basadas en conceptos teóricos. En la contabilidad este tema no ha tenido trascendencia, a pesar de que se habla de los estados financieros clasificados, enfocando tal clasificación a la liquidez o solidez, por objeto social, por naturaleza o función de las operaciones financieras, pero todo a partir de criterios dirigidos solamente a lo financiero. Sin embargo, la contabilidad no se puede reducir a la contabilidad financiera; se requiere mirar más allá de estos intereses y buscar que la contabilidad tenga la capacidad de clasificar el patrimonio ambiental, basada en criterios libres — ningún sesgo e intereses particulares - que permitan representar el medio ambiente con la importancia que merece y que se necesita con el fin de poder tomar las medidas pertinentes para su protección y conservación.

2. La medición: se entiende como una cuantificación dentro de un sistema que podrá establecerse adecuadamente mediante la determinación de parámetros (una unidad de medida), frente a los cuales se debe cuantificar un objeto de medición en el que se deben tener en cuenta diferentes aspectos financieros y no financieros. Si se analiza, se puede constatar que la contabilidad financiera ha excluido el proceso de medición pasando directamente a la valoración. Aunque no es tarea fácil, sí se puede empezar a trabajar cómo es posible hacer una adecuada medición de los elementos que integran el medio ambiente; claro está que debe hacerse en equipo, puesto que todo este tema está en construcción.

3. La valoración: se refiere al proceso de asignar valor a un bien o a un objeto, sin necesidad de constituir un valor financiero o económico, el cual está conformado por factores diferentes. Todavía no sabemos dimensionar el valor de estos activos, a saber, por ejemplo, cómo afecta a la comunidad la tala de un árbol, la pesca indiscriminada, entre otras prácticas que no hemos ni medido ni valorado, pero que están afectando esta casa de todos: la Tierra.

4. Revelaciones: su construcción, relacionada con la contametría, debe hacerse desde otra perspectiva, mediante modelos que permitan diferenciar lo social de lo financiero, para la revelación del patrimonio ambiental en los informes que el contador deberá preparar para informar a todos los interesados e involucrados en el tema, es decir, a todos los integrantes de una comunidad. El ser humano, por naturaleza propia, siempre ha tratado de representar su entorno de diferentes formas; en el caso de lo financiero, ha creado modelos que le permiten demostrar y garantizar la gestión y las posibilidades que tiene el inversor en proyectos diferentes. Tomando estos como ejemplo, la contametría debe elaborar sus propios modelos para revelar todo lo relacionado con lo social, en nuestro caso, modelos para revelar lo ambiental. 
5. La interpretación: es el proceso por el cual un contenido material dado e independiente del intérprete es comprendido o traducido en una nueva forma de expresión. Esta visión, en contexto, orientada a encontrar explicaciones, se relaciona con la semiótica y la hermenéutica.

6. La evaluación y control: proceso que se orienta a determinar mediciones de gestión y resultados, en relación con los objetivos de las organizaciones y los intereses de los usuarios $y$, en general, se constituirán por explicaciones fundamentadas en indicadores determinados por las expectativas convergentes, es decir, las de las organizaciones y las de los diversos usuarios que tienden a ser diferentes por los intereses que comportan. Los agentes sociales tendrán sus propias expectativas sobre información y los procesos contamétricos proporcionan los modelos de evaluación requeridos, combinando elementos de clasificación, medición, valoración, interpretación y revelación con aquellos determinados por los objetivos, intereses y expectativas de los grupos de interés.

Cabe decir que la contametría, inicialmente, se conoció como un complemento de la teoría contable; esta constituye un aspecto tecnológico muy importante de la contabilidad y, en el futuro, puede representar una importante palanca para el desarrollo disciplinar, entendiendo su relación con la contabilidad y la posibilidad de construir herramientas tecnológicas que permitan la construcción de soluciones para todos los interrogantes contables que aún debemos responder (Franco, 1998).

\section{Operacionalización de los elementos contamétricos en el caso de la multinacional Drummond en Colombia}

La compañía Drummond se fundó en 1935. Está ubicada en Birmingham, Alabama. Con sede en Estados Unidos, Drummond se dedica principalmente a la extracción, procesamiento y venta de carbón y sus derivados. La compañía comenzó su producción en Colombia en 1995 y se dedica a exportar toda su producción de carbón térmico. La firma cuenta con reservas probadas y probables de aproximadamente $1900 \mathrm{Mt}$, y su producción actual alcanza los 25Mt/a. La operación colombiana de la compañía incluye las minas Pribbenow y El Descanso (a cielo abierto) —ambas situadas en la cuenca carbonífera Cesar, cerca de La Loma-, Puerto Drummond — un puerto marítimo de aguas profundas en el Mar Caribe, cerca de Santa Marta - y se dedica al transporte de carbón e instalaciones de manipulación. En junio del 2011 la empresa firmó una alianza con el conglomerado japonés Itochu para crear una nueva compañía, Drummond International, que operará todos los activos de dicho país.

La multinacional carbonífera ha sido demandada por lo menos en tres ocasiones por recibir apoyo de grupos paramilitares en Colombia, en medio de violaciones de derechos humanos en contra de la población civil — como asesinatos de líderes sindicales, entre otros- . La justicia norteamericana no ha encontrado pruebas suficientes y ha fallado a favor de la empresa, que ha manifestado que nunca ha apoyado ningún tipo de acción de grupos al margen de la ley. 
En febrero del 2013, el periodista de la revista Semana, Alejandro Arias, reportó con pruebas fotográficas el vertimiento de cientos de toneladas de carbón al mar por parte de la multinacional. Este hecho tuvo lugar en enero del mismo año. A partir de este hecho, el Gobierno nacional de Colombia suspendió temporalmente algunas de las operaciones de la compañía en Santa Marta, lugar donde ocurrieron los hechos.

Para analizar la viabilidad y eficacia de la contametría, a continuación se presenta una aproximación de la aplicación de sus elementos en un caso que en Colombia ha generado diferentes problemáticas — tanto en el aspecto ambiental, como en el económico y el social—, en diferentes regiones de los departamentos de Cesar y Magdalena, debido a las acciones que la multinacional Drummond ha efectuado de acuerdo con sus actividades carboníferas de exploración, explotación, transporte y exportación.

Tomando como referente técnico las publicaciones presentadas por Álvaro Prado, director de Colombia Punto Medio (Prado, 2014) y Sandra Vilardy (Vilardy, 2014), bióloga marina y doctora en ecología y medio ambiente, profesora de la Universidad del Magdalena, los siguientes son los aspectos que fundamentan la complejidad del caso y que son ubicados en los elementos contamétricos, con el objetivo de informar acerca de la complejidad de los hechos sociales analizados. Es importante anotar que el siguiente análisis y presentación del caso Drummond se ubica específicamente en el hecho ocurrido el 13 de enero del 2013, cuando los trabajadores de la multinacional vertieron en la bahía de Santa Marta el carbón para no dejar hundir las barcazas. Además, es importante anotar que esta presentación se hace, estrictamente, desde un punto de vista social, con el fin de ilustrar e informar a la sociedad desde otra óptica sobre estos hechos empresariales, como lo es el ya mencionado que afecta el medio ambiente y el bienestar de la sociedad. Los elementos contamétricos también pueden presentar la información desde lo financiero por medio de modelos, pero esta no es la intención del presente trabajo.

1. Clasificación. Se identifican aspectos relacionados con los siguientes factores: primero, el territorio, en donde se realizan tanto las actividades de extracción como las de transporte de carbón, con sus componentes bióticos y abióticos de ecosistemas terrestres y acuáticos; segundo, la población, teniendo en cuenta los habitantes de los departamentos mencionados y el grupo de trabajadores de la empresa; tercero, la economía, según sus implicaciones en el turismo, la agricultura y la ganadería, en el cálculo de las regalías, en algunas multas impuestas por el gobierno de Colombia y el pago a favor de la Drummond por un fallo emitido por la Cámara de Comercio Internacional en contra del Estado colombiano. 
Tabla 1. Clasificación contamétrica del caso Drummond

\begin{tabular}{llll}
\hline $\begin{array}{c}\text { Factores } \\
\text { de dimensión } \\
\text { Social }\end{array}$ & Categoría & Grupo & \\
\hline & Bióticos & Fauna & Peces, zooplancton \\
Territorio & Flora & Algas, fitoplancton \\
& Abióticos & Minas & Carbón \\
\hline Población & Residentes & Habitantes & Agua, aire, tierra \\
\hline & Trabajadores & Transnacional & Dpto. Cesar \\
\hline Economía & Acumulación & Financiera & Rentabilidad \\
& Distribución & Tributaria & Multas y sanciones, regalías \\
& Responsabilidad & Reducción del turismo, agricultura y ganadería \\
\hline Cultura & Ecológica & Social & Dańos por la contaminación \\
& & Mental & $\begin{array}{l}\text { Inconciencia ecológica de la población, de la empresa y del Estado } \\
\text { colombiano }\end{array}$ \\
\hline
\end{tabular}

Fuente: elaboración propia

La tabla muestra los factores de la dimensión social con categorías, grupos y elementos identificados en el caso del vertimiento de carbón al mar, en la bahía de Santa Marta, por parte de los trabajadores de la multinacional Drummond el pasado 13 de enero del 2013.

2. Medición. Son cuantificables, con respecto al territorio, la desaparición de arroyos y desertización de terrenos de vocación agrícola y ganadera en las áreas donde se realiza la extracción del carbón; la mortandad de peces y especies propias del ecosistema marino, por el derrame de toneladas de carbón, en el área en donde se realiza el cargue del mineral a través de barcazas, como microalgas y pequeños organismos que sirven de alimento a los peces. Adicionalmente, se puede medir la presencia de partículas minerales derivadas del carbón en el agua o en el aire y la cantidad de casos identificados con problemas de salud relacionados en la población y en los trabajadores afectados por enfermedades profesionales y asma ocupacional. También son medibles los efectos por la reducción de la actividad económica en el sector del turismo, agricultura y ganadería, las variaciones en los cálculos de las regalías por el manejo de los datos base o insumos de información, y el total de los valores de las multas que ha pagado la empresa multinacional en los años 2007, 2008 y 2014, y que pagó el Estado colombiano en 2012. 
Tabla 2. Medición contamétrica del caso Drummond

\begin{tabular}{|c|c|c|c|c|}
\hline $\begin{array}{l}\text { Factores } \\
\text { de dimensión } \\
\text { social }\end{array}$ & Categoría & Grupo & Elementos & Medición \\
\hline \multirow{4}{*}{ Territorio } & \multirow{2}{*}{ Bióticos } & Fauna & Peces, zooplancton & Proporciones \\
\hline & & Flora & Algas, fitoplancton & Proporciones \\
\hline & \multirow{2}{*}{ Abióticos } & Minas & Carbón & Nominal \\
\hline & & Vitales & Agua, aire, tierra & Proporciones \\
\hline \multirow{2}{*}{ Población } & Residentes & Habitantes & Con enfermedades respiratorias & Proporciones \\
\hline & Trabajadores & Transnacional & Con asma ocupacional & Proporciones \\
\hline \multirow{3}{*}{ Economía } & Acumulación & Financiera & Rentabilidad & Nominal \\
\hline & \multirow{2}{*}{ Distribución } & Tributaria & Multas y sanciones, regalías & Nominal \\
\hline & & Responsabilidad & Reducción del turismo, agricultura y ganadería & Nominal \\
\hline \multirow[b]{2}{*}{ Cultura } & \multirow[b]{2}{*}{ Ecológica } & Social & Daños por la contaminación & Proporciones \\
\hline & & Mental & $\begin{array}{l}\text { Inconciencia ecológica de la población, de la empresa } \\
\text { y del Estado colombiano }\end{array}$ & Ordinal \\
\hline
\end{tabular}

Fuente: elaboración propia

La tabla adiciona los criterios de medición a los factores de dimensión social, esquematizados con categorías, grupos y elementos que se identificaron en la fase de clasificación. Las proporciones se refieren a porcentajes: calculadas, en el factor territorial, desde las variaciones identificadas en el ecosistema frente a otros similares no afectados y, en la población, según los casos detectados frente al total. La medición nominal se refiere a cantidades numéricas o en dinero, asociadas a cada elemento, mientras que el criterio ordinal facilita la definición de categorías o niveles para ubicar la idiosincrasia de las personas que forman parte de los grupos mencionados.

3. Valoración. Como degradación de los ecosistemas y el medio ambiente, se pueden catalogar la desaparición de fuentes hídricas y la ausencia de acciones para reversar la erosión generada en las áreas de extracción carbonífera; asimismo, la contaminación orgánica e inorgánica que afecta el ecosistema marino y la salud de habitantes y trabajadores, además de la reducción significativa en la pesca artesanal y la imposibilidad de desarrollar actividades económicas de agricultura, ganadería y turismo. El cálculo de las regalías se caracteriza por la falta de transparencia, mientras que las multas que ha pagado la empresa Drummond no son significativas frente a sus ingresos (no superan cuatro mil millones de pesos), mientras que el valor que el Estado colombiano pagó representó un fuerte golpe al Presupuesto General de la Nación (sesenta mil millones de pesos). 
Tabla 3. Valoración contamétrica del caso Drummond

\begin{tabular}{|c|c|c|c|c|c|}
\hline $\begin{array}{l}\text { Factores de } \\
\text { dimensión } \\
\text { social }\end{array}$ & Categoría & Grupo & Elementos & Medición & Valoración \\
\hline \multirow{4}{*}{ Territorio } & \multirow{2}{*}{ Bióticos } & Fauna & Peces, zooplancton & Proporciones & \multirow{4}{*}{ Degradación de los ecosistemas } \\
\hline & & Flora & Algas, fitoplancton & Proporciones & \\
\hline & \multirow{2}{*}{ Abióticos } & Minas & Carbón & Nominal & \\
\hline & & Vitales & Agua, aire, tierra & Proporciones & \\
\hline \multirow[t]{2}{*}{ Población } & Residentes & Habitantes & $\begin{array}{l}\text { Con enfermedades } \\
\text { respiratorias }\end{array}$ & Proporciones & \multirow[t]{2}{*}{$\begin{array}{l}\text { Deterioro en la salud y baja calidad de } \\
\text { vida }\end{array}$} \\
\hline & Trabajadores & Transnacional & Con asma ocupacional & Proporciones & \\
\hline \multirow{3}{*}{ Economía } & Acumulación & Financiera & Rentabilidad & Nominal & \multirow{3}{*}{ Ausencia de valor agregado } \\
\hline & \multirow{2}{*}{ Distribución } & Tributaria & Multas y sanciones, regalías & Nominal & \\
\hline & & Responsabilidad & $\begin{array}{l}\text { Reducción del turismo, } \\
\text { agricultura y ganadería }\end{array}$ & Nominal & \\
\hline \multirow[b]{2}{*}{ Cultura } & \multirow[b]{2}{*}{ Ecológica } & Social & $\begin{array}{l}\text { Daños por la } \\
\text { contaminación }\end{array}$ & Proporciones & Nivel de aculturación o resilencia \\
\hline & & Mental & $\begin{array}{l}\text { Inconciencia ecológica de la } \\
\text { población, de la empresa y } \\
\text { del Estado colombiano }\end{array}$ & Ordinal & Anomia (pérdida de valores) \\
\hline
\end{tabular}

Fuente: elaboración propia

La tabla muestra cómo en la etapa de valoración a los elementos identificados y medidos en las fases anteriores se asignan los juicios que surgen como resultado de la valoración de los insumos de información obtenidos.

4. Revelación. La construcción de la revelación parte de los elementos identificados en la etapa de clasificación, incluyendo su respectiva medición y valoración e integrando datos cuantitativos y cualitativos para hacerlos comprensibles en una matriz o diagrama fácil de entender por los diferentes usuarios de la información, simplificando así la complejidad del caso analizado. El objetivo de la revelación es mostrar el daño ambiental y su impacto, analizando las implicaciones para la empresa Drummond y para el país, evidenciando también los errores en la gestión de varias instituciones colombianas. 
Tabla 4. Representación contamétrica del caso Drummond

\begin{tabular}{|c|c|c|c|c|c|c|}
\hline $\begin{array}{c}\text { Factores de } \\
\text { dimensión } \\
\text { social }\end{array}$ & Categoría & Grupo & Elementos & Evidencia & Diagnóstico & Pronósticos \\
\hline \multirow{4}{*}{ Territorio } & \multirow[b]{2}{*}{ Bióticos } & Fauna & Peces, zooplancton & & $\begin{array}{l}\text { Muerte por asfixia o por } \\
\text { obstrucciones digestivas }\end{array}$ & \multirow{4}{*}{$\begin{array}{l}\text { De continuar con } \\
\text { la contaminación } \\
\text { indiscriminada, muchas } \\
\text { especies desaparecerán y } \\
\text { al agotarse los recursos } \\
\text { no renovables Colombia } \\
\text { perderá competitividad }\end{array}$} \\
\hline & & Flora & Algas, fitoplancton & $\begin{array}{l}\text { Entre el } 12 \text { y } 13 \text { de } \\
\text { enero del } 2013 \text {, derrame }\end{array}$ & $\begin{array}{l}\text { Las microalgas del sustrato } \\
\text { quedan inhibidas para } \\
\text { realizar la fotosíntesis }\end{array}$ & \\
\hline & \multirow{2}{*}{ Abióticos } & Minas & Carbón & $\begin{array}{l}\text { de entre } 500 \text { y } 1800 \\
\text { toneladas de carbón }\end{array}$ & $\begin{array}{l}\text { Agotamiento de los recursos } \\
\text { no renovables }\end{array}$ & \\
\hline & & Vitales & Agua, aire, tierra & & $\begin{array}{l}\text { Contaminación orgánica e } \\
\text { inorgánica }\end{array}$ & \\
\hline \multirow[b]{2}{*}{ Población } & Residentes & Habitantes & $\begin{array}{l}\text { Con enfermedades } \\
\text { respiratorias }\end{array}$ & $\begin{array}{l}\text { Desplazamiento por la } \\
\text { polución }\end{array}$ & $\begin{array}{l}\text { Pobladores de El Hatillo, } \\
\text { Plan Bonito y Boquerón } \\
\text { esperan reubicación desde } \\
2010\end{array}$ & \multirow{2}{*}{$\begin{array}{l}\text { De continuar el } \\
\text { desconocimiento de la } \\
\text { problemática por parte } \\
\text { del Estado, será éste } \\
\text { quien tenga que asumir } \\
\text { la responsabilidad que } \\
\text { le corresponde a la } \\
\text { multinacional, además de } \\
\text { las vidas que se perderán } \\
\text { y la afectación a la calidad } \\
\text { de vida de los colombianos }\end{array}$} \\
\hline & Trabajadores & Transnacional & $\begin{array}{l}\text { Con asma } \\
\text { ocupacional }\end{array}$ & $\begin{array}{l}\text { Más de } 400 \text { trabajadores } \\
\text { y ex trabajadores } \\
\text { con enfermedades } \\
\text { profesionales y asma } \\
\text { ocupacional (sílice } \\
\text { y carbonilla en los } \\
\text { pulmones) }\end{array}$ & $\begin{array}{l}\text { El Ministerio de Salud } \\
\text { no se ha pronunciado } \\
\text { en relación con las } \\
\text { deficiencias del programa } \\
\text { de salud ocupacional de la } \\
\text { multinacional }\end{array}$ & \\
\hline \multirow{3}{*}{ Economía } & Acumulación & Financiera & Rentabilidad & $\begin{array}{l}\text { El } 85 \% \text { de las utilidades } \\
\text { son para la multinacional }\end{array}$ & & \multirow{3}{*}{$\begin{array}{l}\text { Las utilidades provenientes } \\
\text { de la explotación de } \\
\text { carbón se las lleva la } \\
\text { multinacional y el Estado } \\
\text { colombiano se queda con } \\
\text { la contaminación y el } \\
\text { desastre ambiental }\end{array}$} \\
\hline & \multirow[t]{2}{*}{ Distribución } & Tributaria & $\begin{array}{l}\text { Multas y sanciones, } \\
\text { regalías }\end{array}$ & $\begin{array}{l}\text { Regalías equivalen al } \\
15 \% \text { del precio FOB del } \\
\text { carbón exportado. La } \\
\text { Drummond ha pagado a } \\
\text { Colombia cerca de cuatro } \\
\text { mil millones de pesos } \\
\text { en multas y el Estado } \\
\text { colombiano pagó sesenta } \\
\text { mil millones de pesos en } \\
\text { una sanción impuesta por } \\
\text { la Cámara de Comercio } \\
\text { Internacional }\end{array}$ & $\begin{array}{l}\text { La empresa multinacional } \\
\text { se está quedando con las } \\
\text { utilidades provenientes de } \\
\text { la explotación del carbón y } \\
\text { Colombia se queda con el } \\
\text { desastre ambiental }\end{array}$ & \\
\hline & & Responsabilidad & $\begin{array}{l}\text { Reducción del } \\
\text { turismo, agricultura } \\
\text { y ganadería }\end{array}$ & $\begin{array}{l}\text { La contaminación } \\
\text { impide desarrollar estas } \\
\text { actividades económicas }\end{array}$ & & \\
\hline \multirow[b]{2}{*}{ Cultura } & \multirow[b]{2}{*}{ Ecológica } & Social & $\begin{array}{l}\text { Daños por la } \\
\text { contaminación }\end{array}$ & $\begin{array}{l}\text { Ausencia de acciones } \\
\text { colectivas hacia la } \\
\text { protección de los recursos } \\
\text { naturales }\end{array}$ & $\begin{array}{l}\text { Actitud pasiva de la } \\
\text { comunidad frente a las } \\
\text { problemáticas ambientales }\end{array}$ & \multirow{2}{*}{$\begin{array}{l}\text { Facilidad para que las } \\
\text { empresas multinacionales } \\
\text { se apropien de los recursos } \\
\text { naturales colombianos } \\
\text { para su beneficio } \\
\text { económico sin que se } \\
\text { comprometan con la } \\
\text { recuperación ambiental }\end{array}$} \\
\hline & & Mental & $\begin{array}{l}\text { Inconciencia } \\
\text { ecológica de la } \\
\text { población, de la } \\
\text { empresa y del } \\
\text { Estado colombiano }\end{array}$ & $\begin{array}{l}\text { Aprobación ciudadana } \\
\text { de los dańos ambientales } \\
\text { producidos por } \\
\text { la multinacional } \\
\text { Drummond }\end{array}$ & $\begin{array}{l}\text { Ausencia de educación } \\
\text { ambiental y conciencia de } \\
\text { protección y cuidado de los } \\
\text { recursos no renovables }\end{array}$ & \\
\hline
\end{tabular}

Fuente: elaboración propia

En la tabla aparecen las dimensiones temporales de la contametría como herramientas para informar a los diferentes agentes sociales de la problemática generada por el vertimiento de carbón, en la bahía de Santa Marta, por parte de los trabajadores de la mul- 
tinacional Drummond el pasado 13 de enero del 2013. Para cumplir con el objetivo de informar, se puede observar la simplificación de la complejidad del caso analizado.

5. Interpretación. La tragedia ambiental para Colombia será irreversible y aún no es posible cuantificar su impacto o los efectos para las futuras generaciones, pues se desconoce el valor que tiene nuestro patrimonio ambiental y ecológico. Esto se debe a la falta de educación para crear conciencia ciudadana e interés por la conservación de los recursos naturales, tal como lo dice Elinor (2011), Premio Nobel de Economía, 2009. Elinor propone el cuidado de los bienes comunes (medio ambiente) por todos los interesados e involucrados en su cuidado y manejo.

6. Evaluación y control. Ante los delitos ambientales presentes en el caso analizado, en esta etapa del proceso contamétrico es preciso observar los resultados frente a los objetivos de las instituciones responsables del control y vigilancia ambiental (Agencia Nacional de Licencias Ambientales [ANLA], Capitanía de Puertos, Fiscalía General de la Nación, Contraloría General de la República, Instituto de Investigaciones Marinas y Costeras [Invemar], Departamento Administrativo Distrital del Medio Ambiente de Santa Marta [DADMA], Corporación Autónoma Regional del Magdalena [Corpamag], la Procuraduría Delegada para Asuntos Ambientales y Agrarios, la Superintendencia Delegada de Puertos y la Dirección de Asuntos Marinos, Costeros y Acuáticos del Ministerio de Ambiente) y la aplicación de las políticas sobre zonas costeras: Política Nacional Ambiental para el Desarrollo Sostenible de los Espacios Oceánicos y las Zonas Costeras e Insulares de Colombia (2000) y Política Nacional del Océano y los Espacios Costeros (2007).

Según el Atlas Global de Justicia Ambiental, Colombia es el segundo país en el mundo con más conflictos ambientales, lo cual indica que la riqueza en biodiversidad es altamente vulnerable y está en alto riesgo por problemáticas como la analizada en el presente trabajo, por la sequía en el departamento del Casanare durante mes de marzo de 2014, por el conflicto por la construcción de hoteles en el Parque Tairona, por los efectos de las fumigaciones con glifosato, por proyectos madereros en el Chocó, por la desaparición del río Dagua, por el litigio relacionado con el Páramo de Santurbán, entre otros.

Esta propuesta de aplicación de los elementos contamétricos permite generar una respuesta a las necesidades de información sobre problemas tan complejos como el analizado, que son conocidos parcialmente por los diferentes agentes sociales que toman decisiones relacionadas, lo que dificulta el control y la coordinación interinstitucional que permita proteger realmente el patrimonio natural colombiano. Así, la contabilidad demuestra su capacidad de informar más allá de hechos económicos sobre hechos sociales de gran impacto.

Para el contador público no se genera la necesidad de ser omnisapiente sino que debe estar en capacidad de establecer un diálogo interdisciplinario para nutrirse de los referentes técnicos que se deben tener en cuenta en su misión de responder a las necesidades de la información que la sociedad reclama. Por lo tanto, es preciso reiterar que la esencia del contador público no es dedicarse exclusivamente a cumplir regulaciones en materia contable o tributaria, sino que el compromiso por aportar a su profesión debe observar diferentes problemáticas. 
Finalmente, resta por decir que la responsabilidad social del contador frente a la protección del medio ambiente en Colombia tiene un carácter evolutivo, dada la magnitud del problema ecológico que, como se explicó, aun a corto plazo compromete la supervivencia humana misma. Por otra parte, esta misión requiere de un fuerte componente ético, siempre supeditando el interés económico en pro del medio ambiente que, al final, es el futuro de la humanidad, ya que por la intervención de diferentes factores de poder e intereses económicos no siempre se puede contar con la legislación adecuada. Es necesario darle la interpretación pertinente como se dijo anteriormente, y promover como ciudadanos, y como gremio, los cambios necesarios desde la profesión para el verdadero desarrollo sostenible. Este componente ético debe contagiar a las empresas que, a pesar de la mentalidad dominante en la que prima el interés privado, pueden encontrar de una u otra forma el necesario equilibrio entre el fin lucrativo y la conservación del patrimonio más grande e importante de la humanidad: el medio ambiente.

\section{Bibliografía}

1. Barry, F. (1995). Economía ambiental: una introducción. Bogotá: McGraw-Hill.

2. Coase, R. H. (1988). The Problem of Social Cost. Law Chicaro Universy Of Chicago Press, 95.

3. Conceicao de Almeida, M. (2008). Para comprender la complejidad. Hermosillo, México: Multidiversidad Mundo Real Edgar Morin.

4. Elinor, O. (2011). El gobierno de los bienes comunes. México: Fondo de Cultura Económica.

5. Franco, R. (1998). Contabilidad. Pereira: Investigar Editores.

6. Franco, R. (2012). Concepto de contametría. Criterio Libre, 1.

7. Franco Ruiz, R. (2014, enero-junio). Fundamentos ontológicos para la construcción del concepto de contametría. Rev. Cient. Gen. José María Córdova 12(13), 165-190.
8. MacNeill, J., Winsemius, P. y Yakushji, T. (1991). Beyond Interdependence. The Meshing Of The World's Economy And The Earth's Ecology. Oxford University Press.

9. Manrique, A. (2006). Control social ambiental en Bogotá, D.C. Bogotá: Contraloría de Bogotá.

10. Pardo, Á. (18 de febrero del 2014). Drummond, un desastre ambiental, económico y social. Recuperado de http:// www.razonpublica.com/index.php/econom-y-sociedad-temas-29/3571drummond-un-desastre-ambiental-economico-y-social.html

11. Vilardy, S. (18 de febrero de 2014). Drummond y las debilidades de la gestión costera. Recuperado de http://www. razonpublica.com/index.php/regiones-temas-31/3570-drummond-y-las-debilidades-de-la-gestion-costera.html

12. Zapata, J. (1997). Desarrollo sostenible. Bogotá: Librería del Profesional. 